\title{
Two Numerical Studies for Longitudinal Movement of a Canard UAV with Vectored Thrust
}

\author{
*Liviu Dincă, *Jenica-Ileana Corcău and **Dionis Cumpan \\ *Department of Electrical, Energetic and Aerospace Engineering,University of Craiova, Romania \\ ** AEROSTAR S.A. Bacău, Bacău, Romania \\ ldinca@elth.ucv.ro, jcorcau@elth.ucv.ro. , deniscumpan@gmail.com
}

\subsection{6/AUCEE.2021.1.13}

\begin{abstract}
This work presents a study concerning the possibilities to improve the longitudinal dynamic of a canard UAV using vectored thrust. It is followed to harness the advantages of canard configuration of UAV and to obtain further a better longitudinal dynamic, able to fulfil more complex missions than canard UAV without vectored thrust. There are tested two methods. The first uses the UAV polars obtained in XFLR 5 and extended, using literature experience, a little bit over the stall angle. By this method is determined the necessary gain between the elevator steering angle and thrust deflection angle in order to maintain the UAV in landing configuration (flaps down) to the optimum angle of attack for landing. Using this method is studied also the effect of vectored thrust in maneuver, in cruise configuration. Step signal on the elevator, thrust and both commands simultaneously are applied on the UAV without and with vectored thrust and are identified the advantages of vectored thrust in this situation. The second method uses the polars of the UAV components obtained in XFLR5, extended independently up to a little bit above each stall angle. By this way is studied the effect of the vectored thrust on the atack angle in horizontal flight for the UAV in cruise configuration. There are obtained horizontal flight parameters (speed, elevator steering, angle of attack and thrust), when vectored thrust is used. It is followed to obtain results for attack angle as high as possible. Both methods are limited by the results obtained in XFLR 5, that can't determine polars at atttack angles near stall, and for the second method, by the aerodynamic interferences between $\mathrm{UAV}$ components.
\end{abstract}

Cuvinte cheie: metode numerice, mişcare logitudinală, configuraţie UAV, propulsie vectorizată.

Keywords: numerical methods, longitudinal movement, canard UAV, vectored thrust.

\section{EVOLUTION OF AIRCRAFT BUILDING AND MAINTENANCE PROCESS}

UAVs have known a very fast development in the last period, due to technological progress, their applications reaching a wide variety. UAV configurations are very divers and they folds each time to the mission they were designed. For relative short distance and relative small flight speed (a few dozen of $\mathrm{km} / \mathrm{h}$ ) are used frequently in present multi-rotor UAVs with net superior maneuverability than airplane type UAVs [4]. By the other hand, airplane type UAVs have the advantage of a superior aerodynamic efficiency than multi-rotors, so they are better for long range missions with higher speeds and larger payload [1],[7].

An airplane type configuration with a very high aerodynamic efficiency is the canard [2]. By the way, excepting the glider configuration, with a very large span, canard UAVs can reach the higher aerodynamic efficiency for a given mission. This fact is due to lift obtained both on the wing and the horizontal tail, so the aerodynamic efficiency is higher. Nevertheless, canard configuration has some disadvantages [3]. One disadvantage concerns this configuration can't reach high angle of attack. This fact limits both maneuverability performances and takeoff and landing performances. Low angle of attack means low load factor, high take-off and landing speeds and further, longer runways.

This load factor limitation is produced by the higher angle of attack for the horizontal tail than for the wing. In these conditions, horizontal tail reaches first the stall angle and can't produce enough pitch moment to maintain the airplane and as result, the wing, at high angle of attack. In landing configurations, with flaps at maximum steering angle, the problem is more difficult because the horizontal tail, even with a maximum elevator steering, can't compensate the wing dive moment. A solution to substantially increase the pitch moment is to use vectored thrust [6], [11]. By deflecting the thrust, it is possible to obtain larger pitching moment and further to reach higher angle of attack and to perform manoeuvres with higher load factor.

\section{II.CONFIGURATION OF STUDIED UAV}

In this study is considered a configuration similar to other studies, but with some modifications. This configuration is presented in figure 1 . UAV constructive parameters are presented in table 1 .

TABLE 1. UAV CONSTRUCTIVE PARAMETERS

\begin{tabular}{|c|c|}
\hline \multicolumn{2}{|c|}{ Entire UAV } \\
\hline Length $[\mathrm{m}]$ & 2.25 \\
\hline Span $[\mathrm{m}]$ & 2 \\
\hline Height $[\mathrm{m}]$ & 0.4 \\
\hline Weight $[\mathrm{kg}]$ & 7 \\
\hline \multicolumn{2}{|c|}{ Wing } \\
\hline Aerodynamic profile & NACA 64A-212 \\
\hline Span $[\mathrm{m}]$ & 2 \\
\hline Chord in symmetry plane $[\mathrm{m}]$ & 0.25 \\
\hline External chord [m] & 0.125 \\
\hline
\end{tabular}




\begin{tabular}{|l|l|}
\hline Mean aerodynamic chord [m] & 0.194 \\
\hline Sweep angle $\left[{ }^{\circ}\right]$ & -20 \\
\hline Dihedral angle $\left[{ }^{\circ}\right]$ & 0 \\
\hline Setting angle $\left[{ }^{\circ}\right]$ & 0 \\
\hline \multicolumn{2}{|c|}{ Ampenaj horizontal } \\
\hline Aerodynamic profile NACA 64A-212 \\
\hline Span [m] & 1 \\
\hline Chord in symmetry plane [m] & 0.22 \\
\hline External chord [m] & 0.11 \\
\hline Mean aerodynamic chord [m] & 5 \\
\hline Sweep angle $\left[{ }^{\circ}\right]$ & 0 \\
\hline Dihedral angle $\left[{ }^{\circ}\right]$ & 2 \\
\hline \multicolumn{2}{|c|}{ Ampenaj vertical } \\
\hline Aerodynamic profile & NACA 0012 \\
\hline Span [m] & 0.25 \\
\hline Chord in symmetry plane [m] & 0.22 \\
\hline External chord [m] & 0.11 \\
\hline Mean aerodynamic chord [m] & 0 \\
\hline
\end{tabular}

Wing aerodynamic profile was choose NACA 64A212 , with a relative high thickness, good aerodynamic qualities and ensures a good wing rigidity. In order to improve aerodynamic efficiency a negative sweep angle was used. A negative sweep angle also avoids flutter danger. Wing setting angle is $0^{\circ}$.

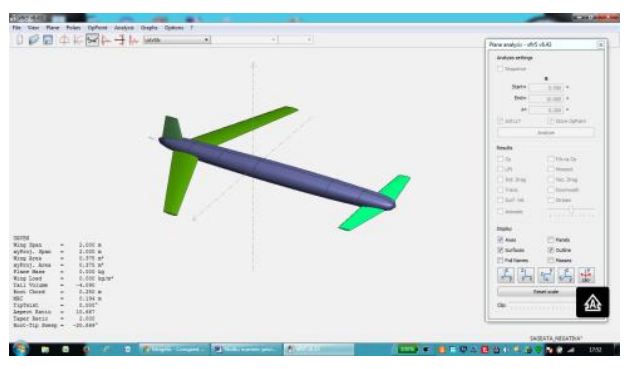

Fig. 1. Studied UAV configuration.

Because the horizontal tail has to produce a big enough pitching moment in order to maintain the longitudinal equilibrium we gave up the practice to use symmetric aerodynamic airfoil and it is adopted also NACA 64A-212 with a setting angle $2^{\circ}$. In these conditions, horizontal tail offers enough lift to maintain longitudinal equilibrium. For the vertical tail it is used the symmetric profile NACA 0012, suited for this application. Using XFLR5 the following polar curves are obtained for this configuration.

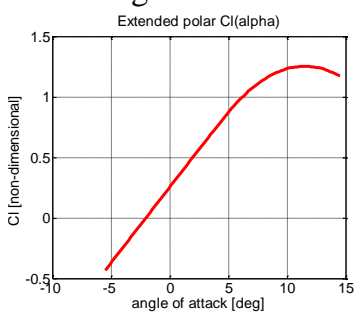

a.

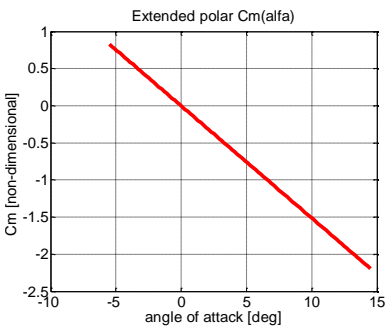

c.

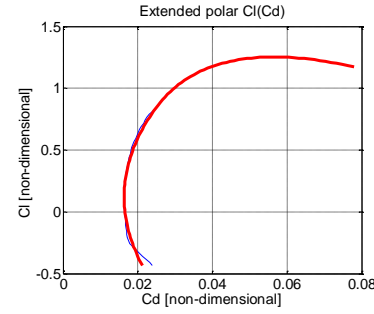

b.

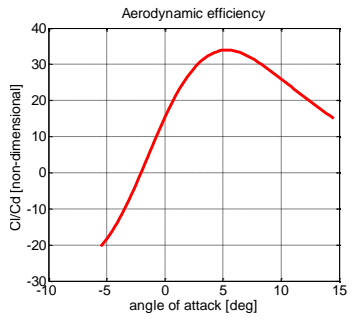

d.
Fig. 2. Polar curves for the studied configuration.
XFLR5 can't determine polar curves at high angle of attack, near the stall angle. For these angle of attack values the polar curves were extrapolated and approximated using profiles data and indications in literature.

We can observe a high aerodynamic efficiency, above 30 , that is a very good value for airplane configuration. Aerodynamic studies were performed neglecting the fuselage, because XFLR5 has a better behavior in these conditions.

From $C_{m}(\alpha)$ dependence it is obtained equilibrium attack angle $0^{\circ}$, with elevator in neutral position. This attack angle offers a lift coefficient 0.278 , useful at high speed.

Take-off and landing conditions were studied also. For take-off it is considered a $20^{\circ}$ flaps steering and for landing $30^{\circ}$. Elevator was considered with a $20^{\circ}$ steering angle both for take off and landing in order to reach the most convenient attack angle. Take-off and landing polars in comparison with cruise polars are presented in figure 3 .
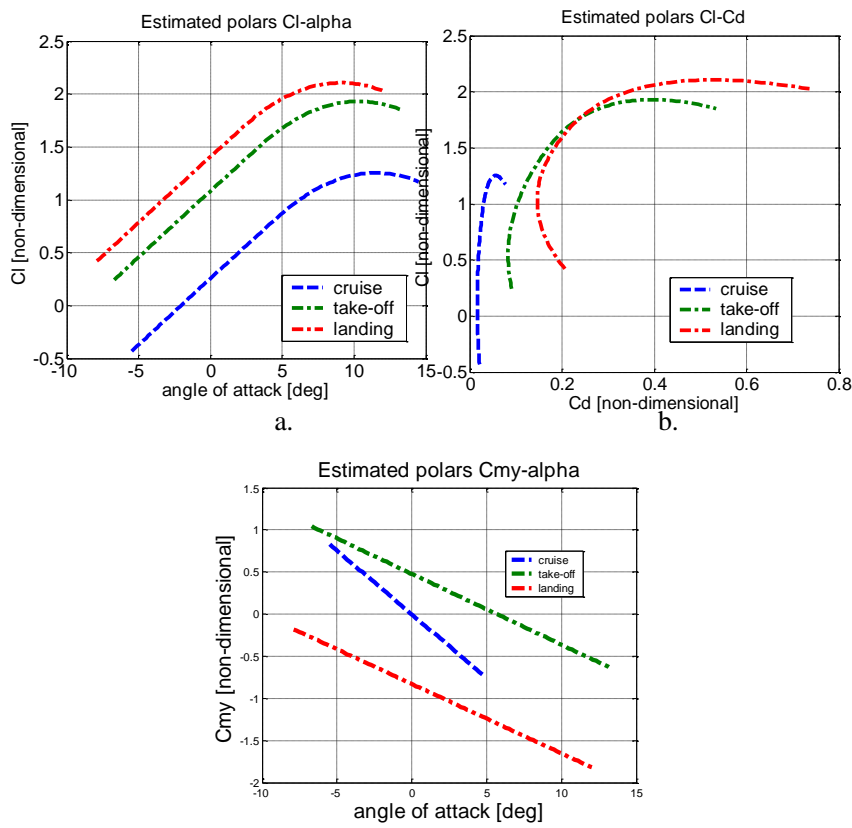

c.

Fig. 3. Take-off and landing polars

In $C_{z}(\alpha)$ polar (figure $3 . \mathrm{a}$ ) we observe a convenient angle of attack both for take-off and for landing about $5^{\circ}$. It is not a high attack angle, but we have to take into account in take-off and landing configuration flaps produces a high dive moment. This angle of attack can be maintained in take-off configuration with a maximum elevator steering angle about $20^{\circ}$, as it results in $C_{m}(\alpha)$ dependence (figure 3.c). For landing configuration, in order to maintain the $5^{\circ}$ angle of attack, it is necessary an extra pitching moment coefficient $\Delta C_{m}=1.24$. This moment can be obtained with vectored thrust. 


\section{IMPROVING LONGITUDINAL BEHAVIOUR WITH VECTORED THRUST}

The aim of this study is to obtain with vectored thrust a pitching moment to maintain the UAV at $5^{\circ}$ angle of attack, considered optimum for landing. A simple hypothesis concerning vectored thrust is to consider the thrust deflection angle proportional with the elevator steering angle. It is produced by this way the extra pitch moment with UAV behavior similar with a UAV with higher elevator efficiency. So, we consider the thrust deflection proportional with the elevator steering and we will follow to reach a $5^{\circ}$ attack angle in landing configuration.

UAV equilibrium with vectored thrust is presented in figure 4 and is described by equations (1) [2],[5].

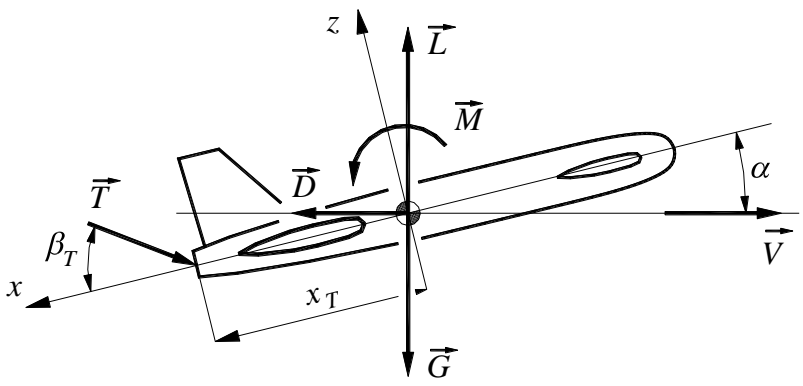

Fig. 4. Longitudinal equilibrium of UAV with vectored thrust.

$$
\left\{\begin{array}{c}
L-G-T \sin \left(\beta_{T}-\alpha\right)=0 \\
D-T \cos \left(\beta_{T}-\alpha\right)=0 \\
M+T \cdot x_{T} \sin \beta_{T}=0
\end{array}\right.
$$

From the second equation results

$$
T=\frac{\frac{\rho}{2} V^{2} S C_{d}}{\cos \left(\beta_{T}-\alpha\right)}
$$

that substituted in the third leads to

$$
\frac{\rho}{2} V^{2} S c_{m a} C_{m}-\frac{\rho}{2} V^{2} S C_{d} x_{T} \frac{\sin \beta_{T}}{\cos \left(\beta_{T}-\alpha\right)}=0
$$

Supposing attack angle $\alpha$ small $\left(5^{\circ}\right)$ it is possible to approximate $\cos \left(\beta_{T}-\alpha\right) \approx \cos \beta_{T}$ and results

$$
\operatorname{tg} \beta_{T}=\frac{c_{m a} \cdot C_{m}}{x_{T} C_{d}}
$$

Relation (4) is very simple and allows determining the thrust deflection to obtain the desired angle of attack. In relation (4) $C_{m}$ and $C_{d}$ are the aerodynamic coefficients for the optimum landing attack angle in landing configuration.

Considering proportionality between thrust deflection and elevator steering

$$
\beta_{T}=K_{T} \cdot \beta_{e}
$$

and elevator steering in landing $\beta_{e}=20^{\circ}$, proportionality coefficient $K_{T}$ can be found.
With the polars considered before results $C_{\text {mland }}=-1.24, C_{\text {xland }}=0.313 C_{\text {zland }}=1.955$. From UAV configuration it is obtained $x_{T}=1.05 \mathrm{~m}$ and so, $\beta_{T}=36.5^{\circ}$, with $K_{T}=1.82$.

Equilibrium angles of attack, without vectored thrust, in cruise configuration can be found from $C_{m}(\alpha)$ dependences for different elevator steering angles, using XFLR5. These are shown in figure 5.a and dependence between elevator steering and equilibrium angle of attack is in figure 5.b. Elevator steering angles were considered between $0^{\circ}$ and $20^{\circ}$ with $5^{\circ}$ step.
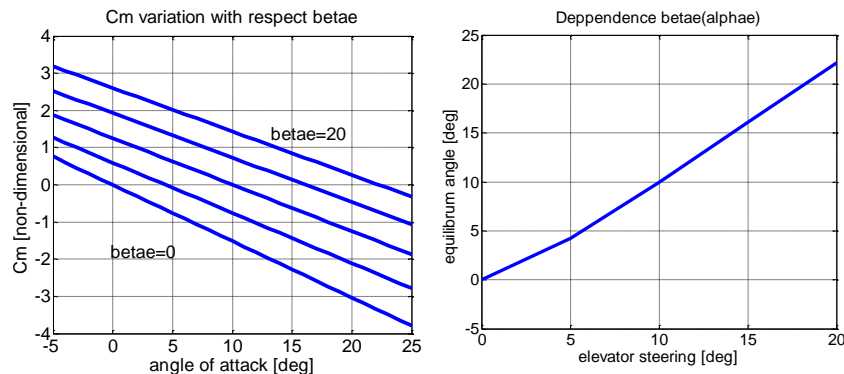

Fig. 5. Equilibrium angle of attack without vectored thrust, cruise configuration.

A convenient dependence between angle of attack and elevator steering is obtained in cruise configuration, so the UAV could be well controlled in cruise configuration.

The same study is performed for landing configuration. Results are in figure 6. Obtained results show high module and negative values for the equilibrium angle of attack. These values were obtained by extrapolation of $C_{m}(\alpha)$ dependences in XFLR5. By consequence, these values have not to be considered accurate, but we can conclude the flaps pitch moment in landing configuration is too large and optimum angle of attack for landing can't be maintained with an acceptable elevator steering.
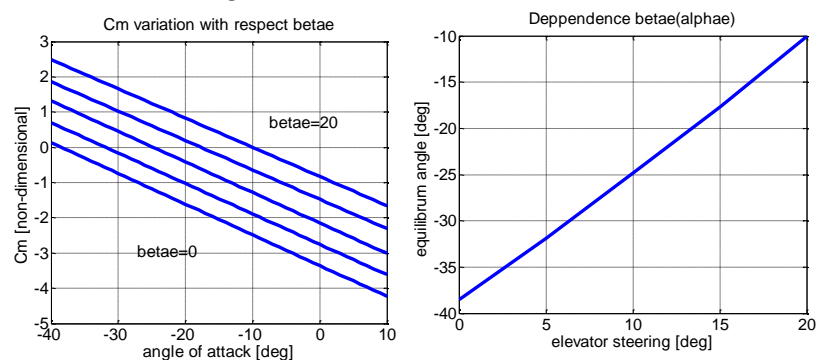

Fig. 6. Equilibrium angle of attack without vectored thrust, landing configuration.

We tried to obtain the optimum landing angle of attack using vectored thrust, with a thrust deflection described by relation (5), with $K_{T}$ values around 1.82, as it resulted from previous estimations.

For this situation, aerodynamic coefficients have to satisfy relation

$c_{m a} \cdot C_{m}\left(\alpha, \beta_{e}\right)-x_{T} C_{d}\left(\alpha, \beta_{e}\right) \frac{\sin \left(K_{T} \beta_{e}\right)}{\cos \left(K_{T} \beta_{e}-\alpha\right)}=0$ 
that is in fact relation (3) detailed for equilibrium angle of attack in landing configuration. Equation (6) is solved for elevator steering between $5^{\circ}$ and $20^{\circ}$ with step $5^{\circ}$ using dependences $C_{m}\left(\alpha, \beta_{p}\right)$ and $C_{d}\left(\alpha, \beta_{p}\right)$ estimated using XFLR 5.

Using these dependences we represented function $F$ in the left hand term in equation (6) and found the equilibrium angles of attack.

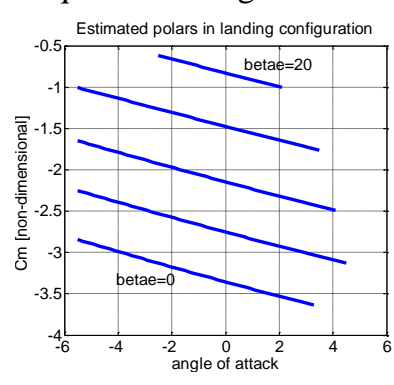

a.

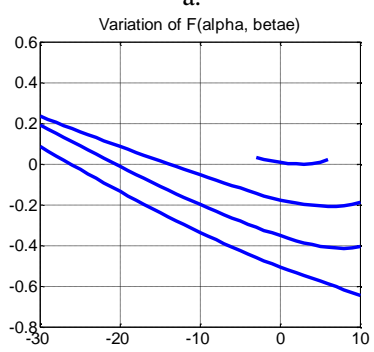

c.
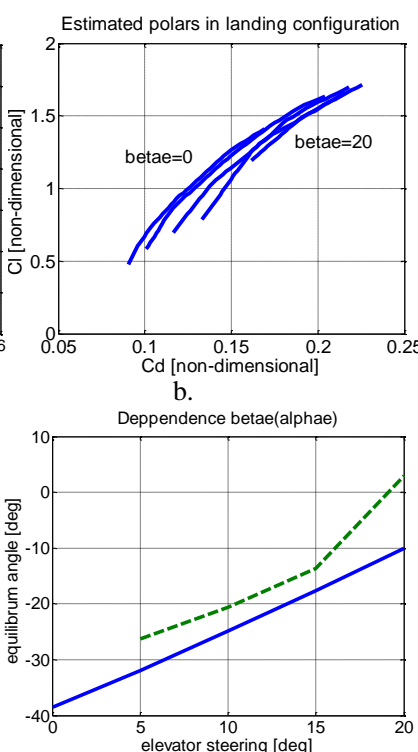

d.
Fig. 7. F function variation and equilibrium angle of attack in landing configuration (dashed line in figure 7.d).

In figure 7.d we observe elevator steering around $20^{\circ}$ can maintain angle of attack around optimum value in landing configuration. Nevertheless, a very rapid decrease of equilibrium angle of attack produces when elevator steering decrease. Again, equilibrium angle of attack lower than $-10^{\circ}$ can not be considered accurate, but we have to notice it is possible to maintain optimum landing angle of attack at maximum elevator steering using vectored thrust.

\section{VECTORED THRUST EFFECTS UPON LONGITUDINAL COMMAND RESPONSE}

In the following is studied the effect of vectored thrust upon the elevator command response, around a flying regime characterized by zero elevator steering and zero thrust deflection, as it assumed in relation (5). In this purpose we used the longitudinal movement equations [5],[8], [9]

$$
\left\{\begin{array}{l}
m \frac{\mathrm{d} v_{x}}{\mathrm{~d} t}=T \cos \left(\beta_{T}\right)-D \cos \alpha-G \sin \alpha \\
m \frac{\mathrm{d} v_{z}}{\mathrm{~d} t}=T \sin \left(\beta_{T}\right)+G \cos \alpha-L \cos \alpha \\
J_{y} \frac{\mathrm{d} \omega_{y}}{\mathrm{~d} t}=M+T \cdot x_{T} \sin \beta_{T}
\end{array}\right.
$$

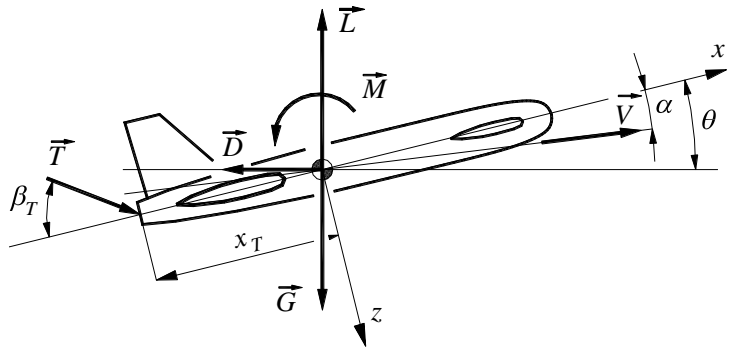

Fig. 8. Reference frame for command response study.

Equations (7) were deduced using the flight dynamics reference frame, as is presented in figure 8.

These equations linearized leads to the same system as the system for UAV without vectored thrust, but with some extra terms in the command matrix.

We considered as command variable the elevator steering and thrust, that is accompanied now by the thrust deflection according relation (5) and thrust variation.

Command matrix without vectored thrust is [10],

$$
B=\left[\begin{array}{cc}
\frac{\partial F_{x}}{\partial \beta_{e}} & \frac{\partial F_{x}}{\partial T} \\
\frac{\partial F_{z}}{\partial \beta_{e}} & \frac{\partial F_{z}}{\partial T} \\
\frac{\partial J_{y}}{\partial \beta_{e}} & \frac{\partial F_{z}}{\partial T} \\
n & 0
\end{array}\right]
$$

and when vectored thrust is used, it becomes

$$
B=\left[\begin{array}{cc}
\frac{\partial F_{x}}{\partial \beta_{e}}-\frac{T}{m} K_{T} \sin \left(K_{T} \beta_{e}\right) & \frac{\partial F_{x}}{\partial T} \\
\frac{\partial F_{z}}{\partial \beta_{e}}-\frac{T}{m} K_{T} \cos \left(K_{T} \beta_{e}\right) & \frac{\partial F_{z}}{\partial T} \\
\frac{\partial J_{y}}{\partial \beta_{e}}+\frac{T}{J_{y}} x_{T} K_{T} \cos \left(K_{T} \beta_{e}\right) & \frac{\partial M_{y}}{\partial T} \\
n & n
\end{array}\right]
$$

Stability and command matrices can be obtained using XFLR5 for airplane without vectored thrust, and for data considered in this study are.

$$
\begin{aligned}
A & =\left[\begin{array}{cccc}
-0.00022 & 0.1601 & 0 & -9.81 \\
-0.3555 & -12.478 & 46.560 & 0 \\
3.287 \cdot 10^{-11} & -1.207 & -1.9087 & 0 \\
0 & 0 & 1 & 0
\end{array}\right] \\
B & =\left[\begin{array}{cc}
0.214 & 9.9 \cdot 10^{-3} \\
-142.15 & 0 \\
10.278 & 0 \\
0 & 0
\end{array}\right]
\end{aligned}
$$

We considered a flying regime in cruise configuration with $0^{\circ}$ elevator steering, with parameters $\mathrm{V}=55.204 \mathrm{~m} / \mathrm{s}, \quad \beta_{e 0}=0^{\circ} \quad, \quad \alpha_{0}=-1.172^{\circ}$, $C_{z 0}=0.098, C_{x 0}=0.016$. Inertial parameters are $\mathrm{m}=7 \mathrm{~kg}, J_{y}=44.72 \mathrm{kgm} 2$ and gravity center position $\mathrm{XCG}=-0.7 \mathrm{~m}$. A SIMULINK study produced results in figure 9. 

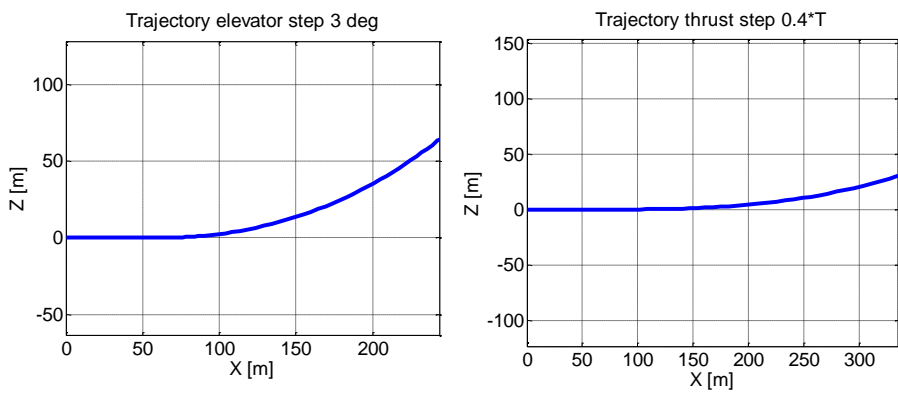

a.

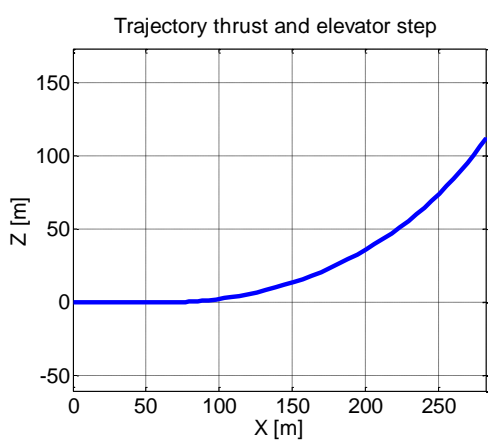

c.

Fig. 9. Command response without vectored thrust.

In the case with vectored thrust, command matrix is (12) and the command response is presented in figure (10).

$$
B=\left[\begin{array}{cc}
-6.108 & 9.901^{-3} \\
-4.517 \cdot 10^{2} & 0 \\
3.354 \cdot 10^{2} & 0 \\
0 & 0
\end{array}\right]
$$

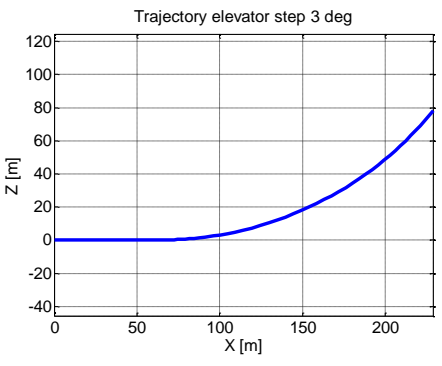

a.

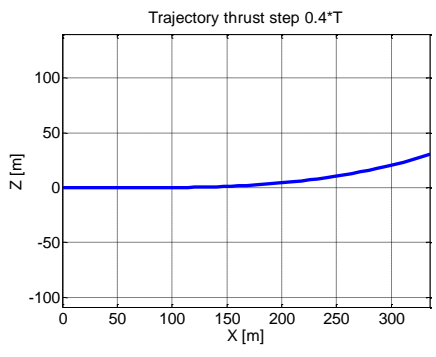

b.

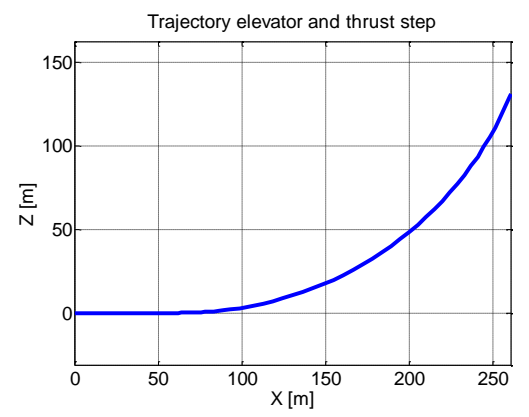

c.

Fig. 10. Command response with vectored thrust.

We can observe about $25 \mathrm{~m}$ extra altitude after 5 second from the step command, when vectored thrust is used. It correspond an extra climbing slope about 5 deg when the elevator steering step is $3^{\circ}$ and thrust deflection is about $6 \mathrm{deg}$. A more edifying evaluation of the vectored thrust effect can be obtained if is estimated parameter $\partial \beta_{e} / \partial n_{z}$ [7] that is often used for airplane maneuverability estimation.

\section{Study Of The Longitudinal Movement Using UAV COMPONENTS POLARS}

Previous study based on the UAV polars obtained in XFLR 5 has an important limit using the UAV angle of attack. XFLR 5 can not compute the polars at high angle of attack. We intent to determine in the following the vectored thrust UAV behavior at high attack angles, near the take-off and landing angles of attack. In this purpose we studied first the polars for each UAV component wing, stabilizer, fin - in XFLR 5. For each component the polars are determined for higher attack angles, even not for attack angles near stall angle. After that, based on the literature experience we performed an estimation of these polars until the stall attack angle and a little bit higher. We assumed the forces upon the UAV as the sum of the forces upon each component. This approach has also limits due to aerodynamic interferences between the UAV components, but we accept this approach in this study.

This study is performed for the cruise configuration. Components polars obtained by the specified method are presented in figures 11,12 and 13.
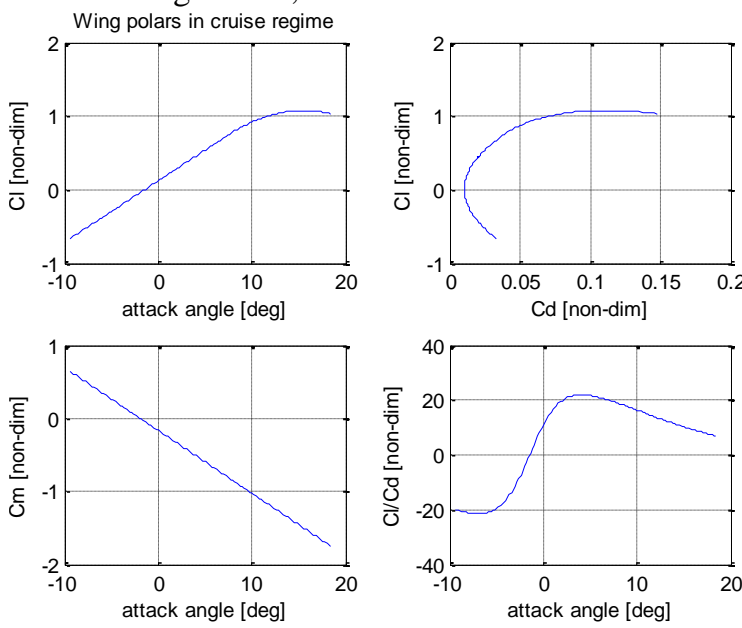

Fig. 11. Wing polars in cruise regime.
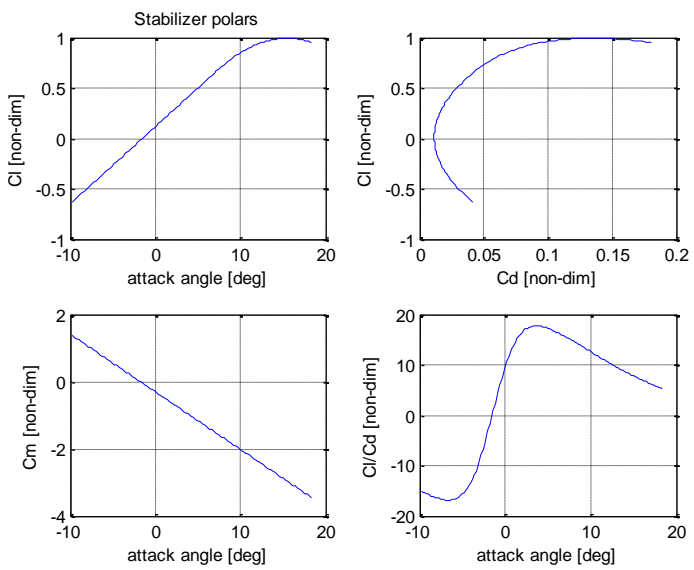

Fig. 12. Stabilizer polars 

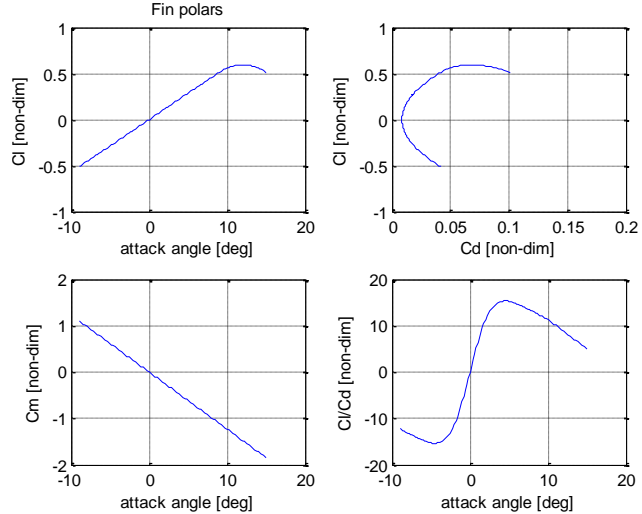

Fig. 13. Fin polars

We studied the horizontal flight at different angles of attack, considering the vectored thrust effects. In this purpose we took into account the following forces and moments upon the UAV: weight; lift, drag and pitching moment due to the wing in translation movement, lift, drag and pitching moment due to the stabilizer in translation movement; forces and moments due to the engine; forces and moments due to the elevator steering. We considered the forces along $\mathrm{x}$ and $\mathrm{z}$ axis and the pitching moment.

\section{FLIGHT PARAMETERS ESTIMATION FOR EQUILIBRIUM CONDITIONS}

In order to determine the equilibrium flight parameters we considered the non-linear system

$$
\left[\begin{array}{c}
F_{x} \\
F_{z} \\
M_{y}
\end{array}\right]=\left[\begin{array}{c}
F_{x}\left(\alpha, \beta_{p}, T\right) \\
F_{z}\left(\alpha, \beta_{p}, T\right) \\
M_{y}\left(\alpha, \beta_{p}, T\right)
\end{array}\right]
$$

with the parameters attack angle $\alpha$, elevator steering $\beta_{p}$ and engine thrust $T$. Between the thrust deflection angle and the elevator steering angle we considered a linear dependence with different proportionality factors with respect the gravity center position. For each flight speed we solved the system (12) using Newton method with a relaxation factor 0.4 . By this way we determined for each horizontal speed the angle of attack, elevator steering and thrust. For four center of gravity positions $X_{c g}=-0,6$, $0,4,-0,2$ and $0 \mathrm{~m}$ we obtained the results in figure 14 .
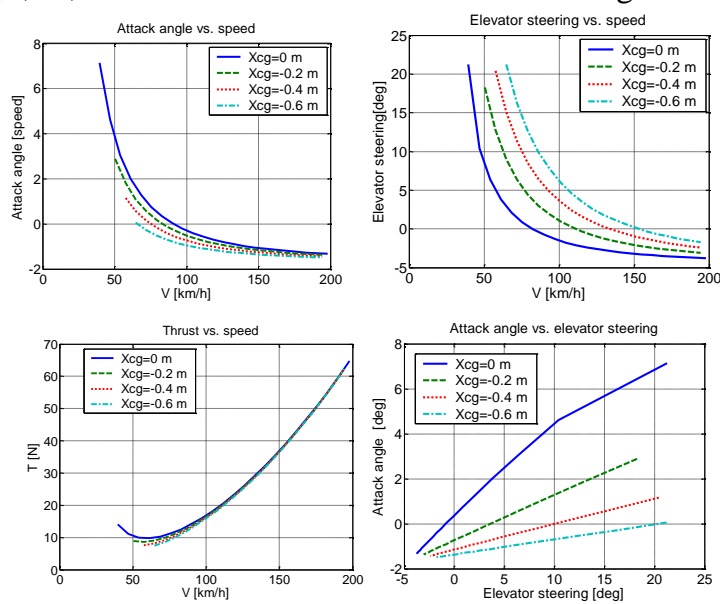

Fig. 14. Flight parameters for each gravity center positing.
As consequence of attack angle limitation once the gravity center moves forward, minimum speed increase for the considered UAV. Thrust variations are negligible.

Further we studied the vectored thrust effect upon the horizontal flight regime. We considered a thrust deflection proportional with the elevator steering. Proportionality factor was considered $K_{T}=2$ for $X_{c g}=-0.2$ and $0 \mathrm{~m}$ and $K_{T}=2$ for $X_{c g}=-0.6$ and $-0.4 \mathrm{~m}$. Engine exhaust was considered first placed rear the airplane as is shown in figure 8. Results for the considered gravity center positions are presented in fgure 15, 16, 17 and 18 .
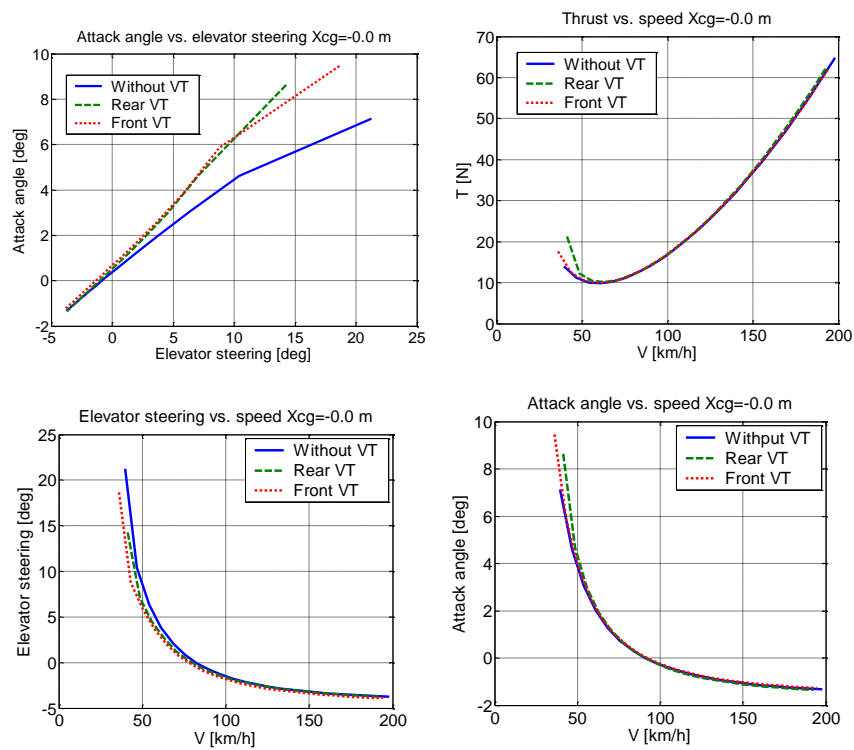

Fig. 15. Flight parameters using vectored thrust for $\mathrm{Xcg}=0 \mathrm{~m}$.
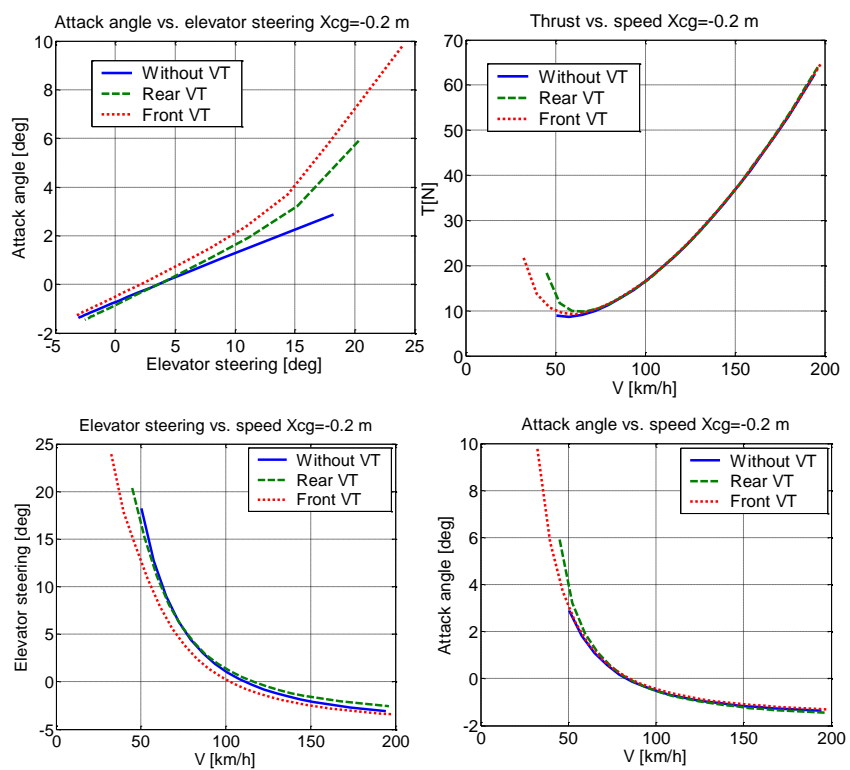

Fig. 16. Flight parameters using vectored thrust for $\mathrm{Xcg}=-0.2 \mathrm{~m}$. 

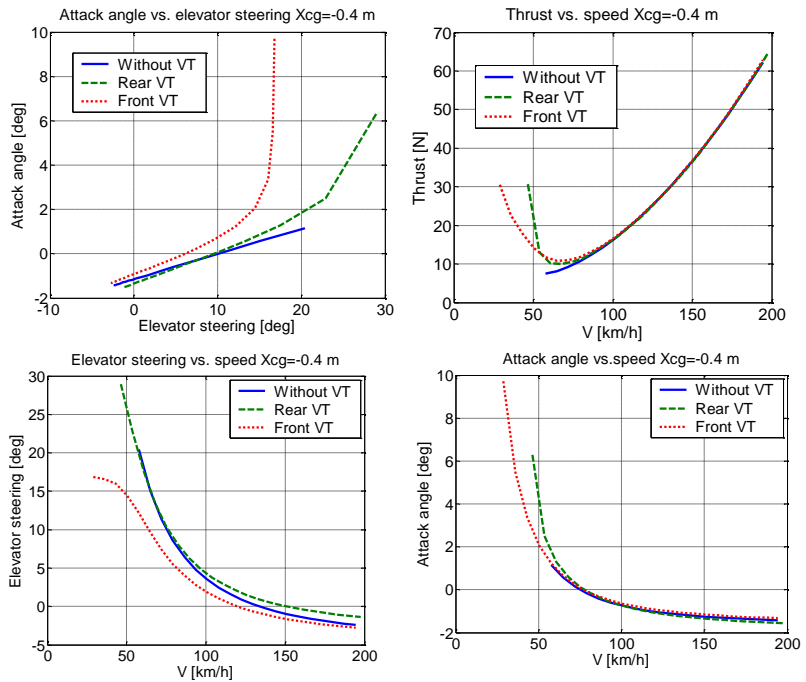

Fig. 17. Flight parameters using vectored thrust for $\mathrm{Xcg}=-0.4 \mathrm{~m}$.
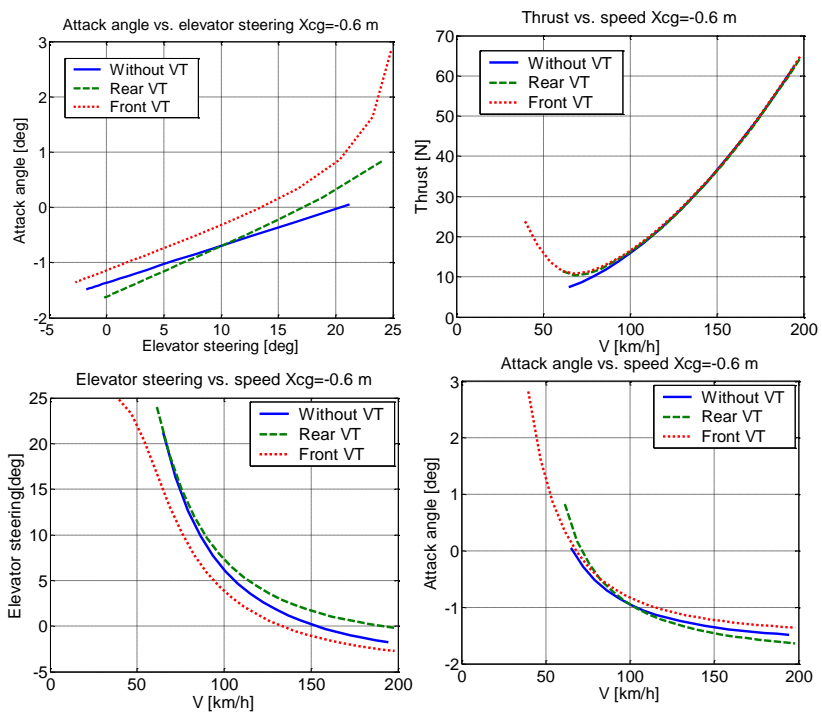

Fig. 18. Flight parameters using vectored thrust for $\mathrm{Xcg}=-0.6 \mathrm{~m}$

Results in figures $15,16,17,18$ show that for the rear placed vectored thrust, efects upos the horizontal flight are negligible concerning the benefits. In order to obtain a pitching moment to help the elevator steering in angle of attack increase and to decrease the minimum speed, thrust has to be deflected downward (see figure 8) and by this way a descending component appears. Even the angle of attack increases and aerodynamic lift increases, the gain is canceled by the descending component of the thrust. Necessary thrust for small speeds increases substantially, but the minimum speed does not decrease significative, even a slight increase in minimal speed appears for a gravity center position $X c g=0 \mathrm{~m}$.

As consequence of this aspect, we can propose a new solution, with two smaller engines placed in front of the UAV, on the stabilizer, that is big enough in this case. For an attack angle increase, now the engines nozzles will be deflected in the opposite direction, so an ascendent component of the thrust will appear and will improve the entire lift of the UAV (see figure 19).

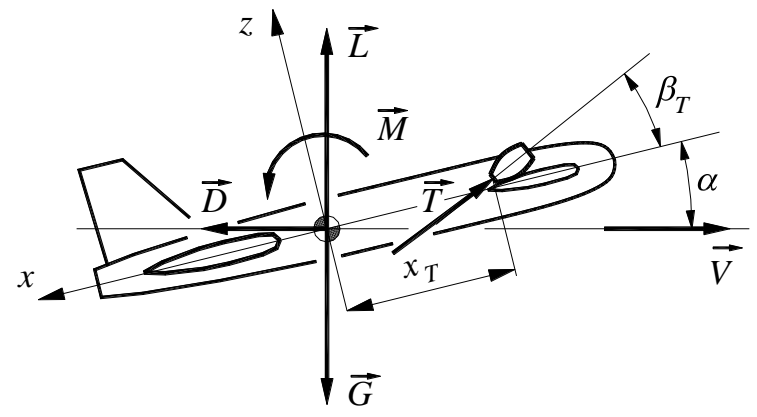

Fig. 19 - Front placed vectored thrust.

We considered in this case also thrust deflections proportional with elevator steering, but gain factors were $K_{T}=1$ for $\mathrm{Xcg}=0 \mathrm{~m}, K_{T}=2$ for Xcg $=-0,2 \mathrm{~m}$ and $K_{T}=3$ for $X c g=-0.4$ and $-0.6 \mathrm{~m}$. Results are presented in comparison with rear placed vectored thrust in figures 15 , 16,17 and 18. This solution offers an increase of attack angle along a semnificative decrease of the minimum speed. For gravity center position $X c g=0 \mathrm{~m}$, minimum speed decrease is small. Here the gain coefficient is small $K_{T}=1$ ), because here the UAV can reach high angles of attack any by elevator steering. Significative decreases of the minimum speed are obtained for the others gravity center positions, where high angles of attack are not accessible without vectored thrust.

For gravity center positions $X c g=-0.4$ and -0.6 , we obtained about a half minimum speed comparing when vectored thrust is not used. So, we combine the advantages of a good static stability with a smaller minimum speed and a better maneuverability, even the gravity center is place far forward.

\section{CONCLUSIONS}

Vectored thrust improves canard UAV maneuverability in all flight phases. It can be useful in take-off and landing configurations in order to obtain optimum angles of attack when flaps are down. Without vectored thrust it is not possible to maintain that angles of attack.

In the paper is presented a simple but efficient method to find the gain coefficient between elevator steering and thrust deflection, when such command law is used. Nevertheless, taking into account results in figure 7 , for landing regime this control law is not very convenient because parameter $\partial \alpha / \partial \beta_{e}$ has a very large value and so is difficult to be control the UAV.

Taking into account initially we followed to improve UAV behavior in landing configuration, it is expected to obtain better results in the control low for thrust deflection angle is considered also the flaps steering angle. In these conditions we could obtain a smaller slope and a more convenient UAV control. In future works it is intended to test control laws with a component proportional with flaps angle and a component proportional with elevator angle. By this way will be improved the behavior in take-off and 
landing configurations, but the effect in the other flying regimes will decrease.

This behavior will be convenient for missions don't need high maneuverability, but only improve take-off and landing qualities to operate on shorter runways. For high maneuverability missions other control laws will be necessary for thrust deflection control, to improve UAV behavior both at take off and in the mission.

Rear positioned vectored thrust has small effects on the horizontal flight performances. Thrust component along oz axis is opposite the airplane lift and even the maximum angle of attack increases, lift increase is canceled by the vertical component of thrust. Minimum horizontal speed does not decrease significative. Vectored thrust advantages maintains in medium and high-speed maneuvers. Torgues produced by the vectored thrust increase the angle of attack and we can obtain higher load factor maneuvers.

A solution to improve the vectored thrust efficiency at small flight speeds is to place the vectored thrust system in front the UAV, in this case two EDF motors placed on the front stabilizer that is large enough in this case. Engines nozzles will be deflected is that way they will produce an ascending component of the thrust, and the gain concerning the minimum speed is significative, mainly for advanced positions of the gravity center.

\section{ACKNOWLEDGMENT}

Source of research funding in this article: Research, program of Electrical, Energetic and Aerospace Engineering Department financed by the University of Craiova.
Contribution of authors:

First author $-40 \%$

Second coauthor $-30 \%$

Third coauthor $-30 \%$

Received on August 05,2021

Editorial Approval on November 30, 2021

\section{REFERENCES}

[1] Dinca, L., Corcau, J.I. - Mechanic and dynamic of airplane flight. Editure Universitaria, Craiova, 2018;

[2] Nita, M. M., Patraulea, R., Sarbu, A., Aircraft mechanic, Reprography IPB, Bucharest, 1985;

[3] Napolitano,M. - Aircraft Dynamics - from modeling to simulation. John Wiley and Sons, 2012;

[4] Marques, P., Da Ronch, A. (editors) - Advanced UAV aerodynamics, flight stability and control: novel concepts, theory and applications. Wiley, 2017;

[5] Hull, D. - Fundamentals of airplane flight mechanics, - Springer Verlag, Berlin, Heidelberg, , 2007;

[6] Vingh, N. - Flight mechanics of high performance aircraft. Cambridge University Press 1993;

[7] Grigore, I. - Airplane flight mechanic - Technical Military Academy, Bucharest 1987;

[8] Kermode, T., Mechanics of flight 11th edition, Pearson Education 2011

[9] Kolk, R., Besserer, C., - Modern flight dynamcs -Literary Licensing LLC 2012;

[10] Stengel, R. - Flight Dynamics - Princeton University Press, 2004;

[11] Dinca L., Corcau J., Numerical Study Concerning Longitudinal Dynamic of a Canard UAV with Vectored Thrust. International Conference on Applied and Theoretical Electricity (ICATE) 2021, 27-28 May, IEEE conference; 10.1109/ICATE49685.2021.9464988; ISBN:978-1-7281-8036-6

[12] Raymer, D. - "Aircraft Design: A Conceptual Approach" - AIAA 2012 